\title{
Study on the Photocatalytic Degradation of Methyl Orange in Water Using Ag/ZnO as Catalyst by Liquid Chromatography Electrospray Ionization Ion-Trap Mass Spectrometry
}

\author{
Tianwen Chen, ${ }^{\text {a,c }}$ Yuanhui Zheng, ${ }^{\mathrm{b}}$ Jin-Ming Lin, ${ }^{\mathrm{a}}$ and Guonan Chen ${ }^{\mathrm{a}}$ \\ ${ }^{a}$ Ministry of Education Key Laboratory of Analysis and Detection Technology for Food Safety (Fuzhou \\ University), and Department of Chemistry, Fuzhou University, Fuzhou, Fujian, China \\ b National Engineering Research Center of Chemical Fertilizer Catalyst, Fuzhou University, Fuzhou, Fujian, \\ China \\ 'Analytical and Testing center, Fuzhou University, Fuzhou, Fujian, China
}

A nanocrystal catalyst $\mathrm{Ag} / \mathrm{ZnO}$ was successfully synthesized using a simple solvothermal method in this study. This catalyst was characterized by X-ray diffraction (XRD) and transmission electron microscopy (TEM). The results showed that this catalyst was composed of metallic $\mathrm{Ag}$ and $\mathrm{ZnO}$. The photodegradation of methyl orange (MO) was investigated in aqueous suspension containing $\mathrm{Ag} / \mathrm{ZnO}$ catalyst under UV irradiation. Liquid chromatography coupled with electrospray ionization ion-trap mass spectrometry was applied to the analysis of the samples coming from the photocatalytic degradation of MO. The experimental results showed that there were four intermediates existing in the photocatalytic reaction. MO could be mineralized in the $\mathrm{Ag} / \mathrm{ZnO}$ suspension after 60 min illumination. (J Am Soc Mass Spectrom 2008, 19, 997-1003) @ 2008 American Society for Mass Spectrometry

$\mathrm{I}$ $\mathrm{n}$ the past decades, photocatalytic techniques have been shown to constitute one of the most promising 1 processes for the wastewater treatment because of its advantages over the traditional techniques, such as quick oxidation, no formation of polycyclic products, and oxidation of pollutants up to the parts per billion (ppb) level. Methyl orange (MO) is an azo dye and has been widely used in textiles, foodstuffs, paper, and leather industries. However, the release of $\mathrm{MO}$ and its products in the environment cause serious pollution problems. The photocatalytic treatment of wastes containing dyes has also been widely reported [1-7]. ZnO has attracted much attention with respect to the degradation of various pollutants because of its high photosensitivity, stability, and wide band gap. Previous studies have proved that $\mathrm{ZnO}$ can degrade some organic pollutants under UV irradiation [8-11]. However, the fast recombination rate of the photo-generated electron and hole pairs may decrease the photocatalytic activity. The combination of transition metals (such as silver, platinum, and iron) and semiconductor has been reported to improve the charge-transfer and photocatalytic activity [12, 13]. However, to the best of our knowledge, there were only a few published reports on

Address reprint requests to Prof. Guonan Chen, Fuzhou University, Department of Chemistry, 523 Gongye Road, Fuzhou, Fujian 350002, China. E-mail: gnchen@fzu.edu.cn
$\mathrm{Ag} / \mathrm{ZnO}$ photocatalysts [14, 15]. They reported the photochemical degradation of lignin, dye, and paper effluent by using Ag-doped $\mathrm{ZnO}$ photocatalysts. The degradation process was monitored by UV-Vis spectrometry in the works. Although the residual organic pollutants could be determined by UV-Vis spectrometry, it was difficult to identify the degradation intermediates.

The intermediates of dyes may be more toxic than the parent compound. Therefore, it is necessary to identify the degradation products. Recently, liquid chromatography separation techniques coupling with mass spectrometry (LC-MS) has been established for separation and detection of dyes and their degradation products [16-20]. In connection with soft ionization techniques, ion-trap mass spectrometry is essential for identification and structural elucidation of dyes.

Some photocatalysts have been used in the photocatalytic degradation of MO [1-4], although the photocatalytic activity of these catalysts was limited and the degradation products were not investigated in detail. The aim of our work is to synthesize the $\mathrm{Ag} / \mathrm{ZnO}$ nanocatalysts with higher photocatalytic activity, and MO was adopted as a compound to evaluate the photocatalytic performance of $\mathrm{Ag} / \mathrm{ZnO}$ catalyst. $\mathrm{MO}$ has poor stability and low volatility, and we considered that liquid chromatography coupling with ion-trap mass spectrometry (LC-IT-MS) was a suitable analytical approach for the identification and determination of 
methyl orange and its degradation products. Therefore, it is innovative for us to synthesize the nanostructured $\mathrm{Ag} / \mathrm{ZnO}$ photocatalysts using a solvothermal method; the residual MO and degradation products are determined by LC-IT-MS. The photocatalytic activity of $\mathrm{Ag} / \mathrm{ZnO}$ photocatalysts provides a simple and effective technique for wastewater treatment. In the present work, the photocatalytic degradation of MO dye under UV irradiation was investigated over nanosized Ag/ $\mathrm{ZnO}$ photocatalysts. We expect that this photocatalytic technique can be applied to the treatment of wastewater in the future.

\section{Experimental}

\section{Chemicals}

HPLC-grade acetonitrile was purchased from Merck (Darmstadt, Germany). Methyl orange (4-[[(4-dimethylamino) phenyl]-azo] benzenesulfonic acid sodium salt) and ammonium acetate (Shanghai Chemical Reagents, Shanghai, China) was analytical reagent-grade. Distilled water was deionized in a Milli-Q SP Reagent Water System (Millipore, Bedford, MA, USA). All the solvents were passed through a $0.45 \mu \mathrm{m}$ cellulose filter before use.

\section{Liquid Chromatography-Mass Spectrometry}

An Agilent 1100 series LC system (Agilent Technologies, Palo Alto, CA, USA) was performed with a binary pump, $1100 \mathrm{UV}-\mathrm{V}$ is diode array detector, an autosampler, and a column thermostat. The LC-MS system was equipped with a Zorbax $C_{18}$ column $(150 \times 4.6 \mathrm{~mm}$ i.d., $5 \mu \mathrm{m})$. The solvent used as mobile phase was acetonitrile: $0.01 \mathrm{M}$ ammonium acetate $(\mathrm{pH}$ 6.8) $=30: 70$ ( vol/vol). The flow rate was $0.6 \mathrm{~mL} \mathrm{~min}^{-1}$, and $20 \mu \mathrm{L}$ of standard or sample solution was injected.

The isocratic HPLC separation was coupled with LC/MSD Trap XCT ion-trap mass spectrometer (Agilent Technologies). The mass spectrometer was equipped with an electrospray ionization (ESI) source and operated at negative polarity. The ESI conditions were as follows: capillary voltage, $3.5 \mathrm{kV}$; the endplate offset was fixed at $-500 \mathrm{~V}$; capillary exit, $100 \mathrm{~V}$; nebulizer pressure, $40 \mathrm{psi}$; drying gas flow, $10 \mathrm{~L} \mathrm{~min}^{-1}$; temperature, $350{ }^{\circ} \mathrm{C}$. The mass range was from 100 to $400 \mathrm{~m} / \mathrm{z}$.

\section{Synthesis}

$\mathrm{Ag} / \mathrm{ZnO}$ nanocatalyst was synthesized by a simple solvothermal method described by Zheng et al. [21]. A certain concentration of $\mathrm{Zn}(\mathrm{Ac})_{2} \cdot 2 \mathrm{H}_{2} \mathrm{O}$ and $\mathrm{CH}_{3} \mathrm{COOAg}$ was mixed in a Teflon tank of $50 \mathrm{~mL}$ capacity. Then, $30 \mathrm{~mL}$ of a $\mathrm{NaOH} /$ ethanol solution $(0.17$ $\mathrm{M})$ was added into the tank drop by drop with agitation. The tank was moved to a stainless steel autoclave, put into an oven, and heated at $160^{\circ} \mathrm{C}$ for $24 \mathrm{~h}$. When the reactions were completed, the autoclave was naturally cooled to room temperature. The brown-red precipitates were collected by filtration, washed with deionized water and ethanol several times, and finally dried in the air at $60{ }^{\circ} \mathrm{C}$ for $10 \mathrm{~h}$.

\section{Degradation Experiment}

For degradation experiment, $30 \mathrm{mg}$ of the catalyst was suspended in $90 \mathrm{~mL}$ of a standard methyl orange (MO) aqueous solution $\left(5.0 \times 10^{-5} \mathrm{M}\right)$, and then the mixture was charged to a quartz tube and agitated overnight in the absence of light. UV irradiation was carried out using a $4 \times 4 \mathrm{~W}$ fluorescent $\mathrm{Hg}$ lamp (Philips TUV $4 \mathrm{~W}$ with the maximum emission wavelength at $254 \mathrm{~nm}$ ). Light intensity near the solution surface was about 150 $\mathrm{mW} / \mathrm{cm}^{2}$. After a given irradiation time, about $3.5 \mathrm{~mL}$ of the mixture was withdrawn and the catalysts were separated from the suspensions by filtration through $0.22 \mu \mathrm{m}$ cellulose membranes.

\section{Results and Discussion}

\section{Catalytic Characterization of $\mathrm{Ag} / \mathrm{ZnO}$}

The X-ray diffraction (XRD) pattern of the synthesized sample is shown in Figure 1a. It can be seen that there are two sets of strong diffraction peaks in Figure 1a, indicating that the synthesized product is a composite material with high crystallinity. Those marked with "\#" can be indexed to hexagonal wurtzited $\mathrm{ZnO}$ (JCPDS file no. 36-1451), whereas the others marked with "** can be indexed to face-center-cubic (fcc) metallic Ag (JCPDS file no. 04-0783), and no other crystalline impurities were observed.

To obtain detailed information about the microstructure and morphology of the synthesized sample, transmission electron microscopy (TEM) was carried out. The result is shown in Figure 1b. A low-magnification TEM image of this sample (see Figure $1 \mathrm{~b}$ ) shows a high yield of $\mathrm{Ag} / \mathrm{ZnO}$ hetero-structure nanocrystals consisting of metallic Ag nanoparticles (highlighted by circles) and $\mathrm{ZnO}$ nanorods. Most of the $\mathrm{Ag}$ nanoparticles and $\mathrm{ZnO}$ nanorods in Figure $1 \mathrm{~b}$ are about $9 \mathrm{~nm}$ in diameter and $18 \mathrm{~nm}$ in width, respectively. Additionally, one can see that all nanoparticles are attached to nanorods. Although there are some $\mathrm{ZnO}$ nanorods without $\mathrm{Ag}$ nanoparticles on the surface, self-nucleated and isolated silver nanoparticles are hardly observed. Moreover, Ag aggregates are also not found in our TEM observations, indicating that all metallic Ag nanoparticles are well dispersed in $\mathrm{ZnO}$ nanorods.

\section{Control Experiments for the Photodegradation}

Preliminary control experiments conducted in the absence of $\mathrm{Ag} / \mathrm{ZnO}$ catalyst showed that less than $1.0 \%$ $\mathrm{MO}$ was decomposed after $1 \mathrm{~h}$ UV irradiation, indicating that $\mathrm{MO}$ has a good photostability under the UV 


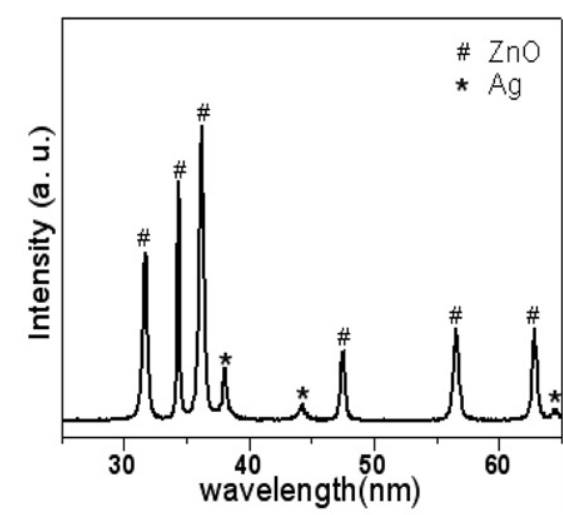

(a)

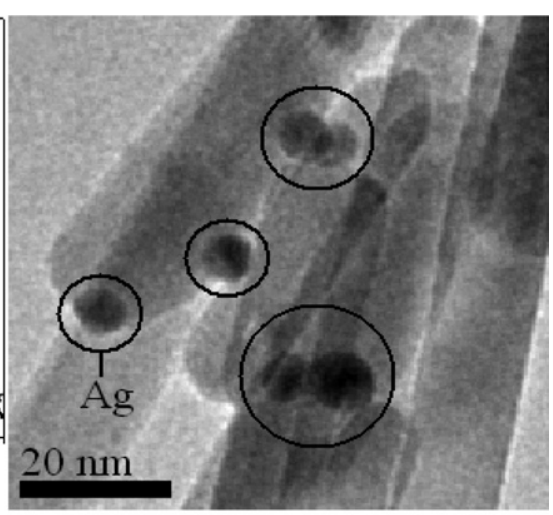

(b)

Figure 1. (a) XRD pattern; (b) TEM image of the as-synthesized Ag/ZnO nanocatalysts.

light irradiation. When there was no UV light irradiation, the concentration of $\mathrm{MO}$ with the addition of $\mathrm{Ag} / \mathrm{ZnO}$ catalyst kept stable in $1 \mathrm{~h}$. Therefore, UV light irradiation and catalyst are the necessary factors in the photocatalytic process.

\section{Determination of MO Residues}

The fragment ions of $\mathrm{MO}$ are produced by tandem mass spectrometry (MS/MS) experiments, where the MO precursor ion is isolated first by the mass analyzer, then the collision activation of this ion is performed yielding the fragment ions. The MS/MS of MO increases the specificity of detection because there is no uncertainty in the product ion spectrum.

The parent molecule of methyl orange provides a signal corresponding to a negative ion at $\mathrm{m} / \mathrm{z} 304[\mathrm{M}-$ $\mathrm{H}]^{-}$[22]. The MS/MS spectrum of this ion gives three significant $m / z$ values: 289.0, 240.2, and 156.1 (see Figure 2 ). The ion at $m / z 289.0$ was derived from the cleavage of one methyl group. The ion at $m / z 240.2$ was derived from the cleavage of a sulfonate group. The ion at $\mathrm{m} / \mathrm{z}$ 156.1 corresponds to $\left[\mathrm{M}-\mathrm{H}-\mathrm{N}_{2} \mathrm{C}_{6} \mathrm{H}_{4} \mathrm{~N}\left(\mathrm{CH}_{3}\right)_{2}\right]^{-}$. The sum of MS/MS product ions at $m / z$ 289.0, 240.2, and 156.1 was for the determination of $\mathrm{MO}$ residues.

Figure 3 shows the change of $\mathrm{MO}$ residues in the presence of $\mathrm{Ag} / \mathrm{ZnO}$, which is used as the catalyst under irradiation. The plot of $\ln \left(\mathrm{C}_{0} / \mathrm{C}\right.$ ) (where $\mathrm{C}_{0}$ is initial concentration of the methyl orange and $C$ is the concentration at a given moment of time) against irradiation time shows that the photocatalytic degradation of methyl orange is a pseudo-first-order reaction. The confirmation of the first-order rate kinetics was derived from the linearity of the plot. The rate constant for degradation of $\mathrm{MO}$ is $1.9 \times 10^{-1} \mathrm{~min}^{-1}$.

\section{Determination of the Photodegradation Products}

Figure 4 shows the total ion chromatograms of methyl orange being degraded for $0,10,20,30,40,50$, and 60 min, respectively. As can be seen from Figure 4 that for $0 \mathrm{~min}$, there are three small peaks of $\mathrm{m} / \mathrm{z} 196,290$, and 320 , except for a strong peak at $\mathrm{m} / \mathrm{z} 304$ corresponding to MO. The peak of $m / z 196$ always exists in the course of photodegradation of $\mathrm{MO}$ for $60 \mathrm{~min}$; perhaps it belongs to the impurity in the MO solution. The peak of

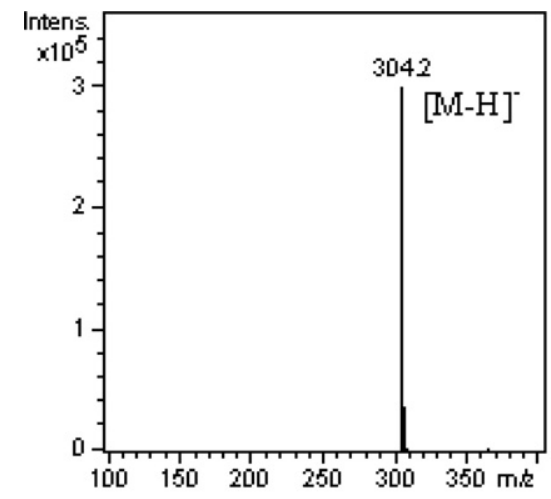

(a)

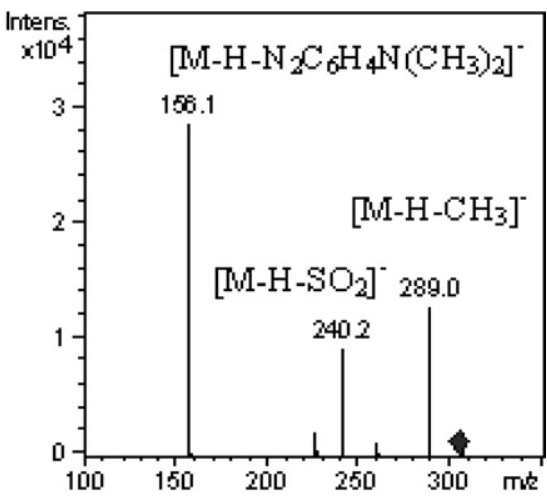

(b)

Figure 2. (-) ESI mass spectra of methyl orange: (a) first-order spectrum, (b) MS/MS spectrum of $\mathrm{m} / \mathrm{z} 304$. 


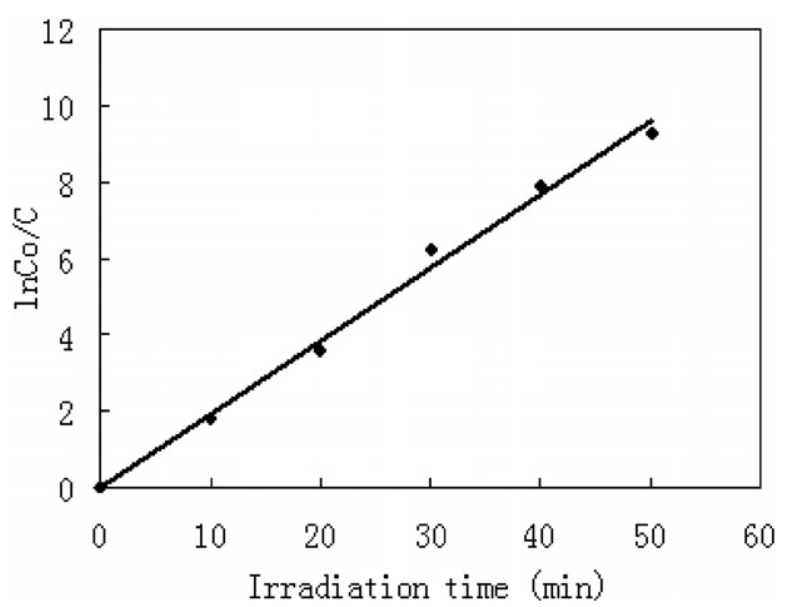

Figure 3. Pseudo-first-order kinetics for decolorization of methyl orange.

$\mathrm{m} / \mathrm{z} 290$ can be attributed to the loss of one methyl group from MO (see Figure 8 below). The MS/MS spectrum of ion at $\mathrm{m} / \mathrm{z} 290$ yields the fragment ions corresponding to $\left[\mathrm{M}-\mathrm{H}-\mathrm{SO}_{2}\right]^{-}$at $m / z 226,[\mathrm{M}-\mathrm{H}$ $\left.-\mathrm{CH}_{3}\right]^{-}$at $\mathrm{m} / \mathrm{z} 275$, and $\left[\mathrm{M}-\mathrm{H}-\mathrm{N}_{2} \mathrm{C}_{6} \mathrm{H}_{4} \mathrm{NHCH}_{3}\right]^{-}$at $\mathrm{m} / \mathrm{z} 156$ (see Figure 5b). The compound of $m / z 320$ is the monohydroxylated product of methyl orange (see Figure 8). The MS/MS spectrum of ion at $m / z 320$ yields the fragment ions corresponding to $\left[\mathrm{M}-\mathrm{H}-\mathrm{SO}_{2}\right]^{-}$at $\mathrm{m} / \mathrm{z}$ 256 and $\left[\mathrm{M}-\mathrm{H}-\mathrm{N}_{2} \mathrm{C}_{6} \mathrm{H}_{3}(\mathrm{OH}) \mathrm{N}\left(\mathrm{CH}_{3}\right)_{2}\right]^{-}$at $\mathrm{m} / z 156$ (see Figure $5 \mathrm{~d}$ ). Since these peaks exist in the samples before UV irradiation, they may be attributed to the limited degradation of $\mathrm{MO}$ by the room light during the LC-MS operating procedures.

After 10 min irradiation, it is found that two new peaks $(\mathrm{m} / \mathrm{z} 276$ and 306$)$ are clearly observed (see Figure 4). The parent molecule with $m / z 304$ loses two methyl groups corresponding to $\mathrm{m} / \mathrm{z} 276$ (see Figure 8). The MS/MS spectrum of ion at $\mathrm{m} / \mathrm{z} 276$ yields the fragment ions corresponding to $\left[\mathrm{M}-\mathrm{H}-\mathrm{SO}_{2}\right]^{-}$at $m / z 212$ and $\left[\mathrm{M}-\mathrm{H}-\mathrm{N}_{2} \mathrm{C}_{6} \mathrm{H}_{4} \mathrm{NH}_{2}\right]^{-}$at $m / z 156$ (see Figure 5a). The compound of $\mathrm{m} / \mathrm{z} 306$ can be attributed to the oxidation in the aromatic ring and loses one methyl group from nitrogen atom of amino group (see Figure 8). The MS/MS spectrum of ion at $m / z 306$ yields the fragment ions corresponding to $\left[\mathrm{M}-\mathrm{H}-\mathrm{SO}_{2}\right]^{-}$at $m / z 242$ and $\left[\mathrm{M}-\mathrm{H}-\mathrm{N}_{2} \mathrm{C}_{6} \mathrm{H}_{3}(\mathrm{OH}) \mathrm{NHCH}_{3}\right]^{-}$at $m / z 156$ (see Figure $5 \mathrm{c}$ ).

As can been seen from Figure 4 that after $20 \mathrm{~min}$ irradiation, $\mathrm{MO}$ and its degradation products exhibit much smaller peaks compared with those after $10 \mathrm{~min}$ irradiation. MO scarcely exists after 30 min irradiation, but some degradation products are still present at this time. After 40 and $50 \mathrm{~min}$ irradiations, degradation products of MO subsequently decrease without formation of new products. We did not find any degradation products through the LC-MS technique after $60 \mathrm{~min} \mathrm{UV}$ irradiation. The photocatalytic activity of $\mathrm{Ag} / \mathrm{ZnO}$ catalyst is much higher than that of other catalysts, such as $\mathrm{TiO}_{2}, \mathrm{Ag} / \mathrm{InVO}_{4}-\mathrm{TiO}_{2}, \mathrm{Ag} / \mathrm{SiO}_{2}, \mathrm{CdS}$, and $\mathrm{Fe} / \mathrm{TiO}_{2}[1$, 23-27]. They reported that MO still existed in the solution after $60 \mathrm{~min}$ UV irradiation. MO did not exist in the solution after $60 \mathrm{~min} \mathrm{UV}$ irradiation with $\mathrm{Ag} /$ $\mathrm{ZnO}$ catalyst.

\section{UV Absorbance Spectra of the Photodegradation Intermediates}

Figure 6 shows the UV spectra for the compounds with molecular weights $\mathrm{MW}=276,290,306,304$, and 320 determined on the basis of $[\mathrm{M}-\mathrm{H}]^{-}$ions in the spectra. The UV-Vis spectrum of the compounds with molecular weights MW $=276$ and 290 shows that the maximum absorption wavelength is a slight blue-shift compared with that of $\mathrm{MO}$, indicating that there is still a chromophore group in this intermediate. Considering that the methyl group can easily separate from the dimethylamine group, we think that products with molecular weights MW $=276$ and 290 are produced by homolytic breaking of the nitrogen-carbon bond, resulting in the substitution of the methyl group by the hydrogen atom.

The UV-Vis spectrum of the compound with molecular weights $\mathrm{MW}=320$ shows a significant red-shift compared with that of MO. This red-shift may be attributed to the hydroxyl radical inserted in the ortho position of the benzene ring linking with the dimethylamine group of MO. A red-shift of the maximal absorption peak of UV-Vis spectra was observed because a hydroxyl group connected with the chromophore group. On the contrary, the dealkylation could lead to the significant wavelength blue-shift. The UV-Vis spectrum of the compound with molecular weights MW = 306 shows a significant blue-shift compared with that of the compound with molecular weights $\mathrm{MW}=320$, because the compound with molecular weights $\mathrm{MW}=$ 306 can be attributed to the loss of one methyl group from the compound with molecular weights $\mathrm{MW}=320$.

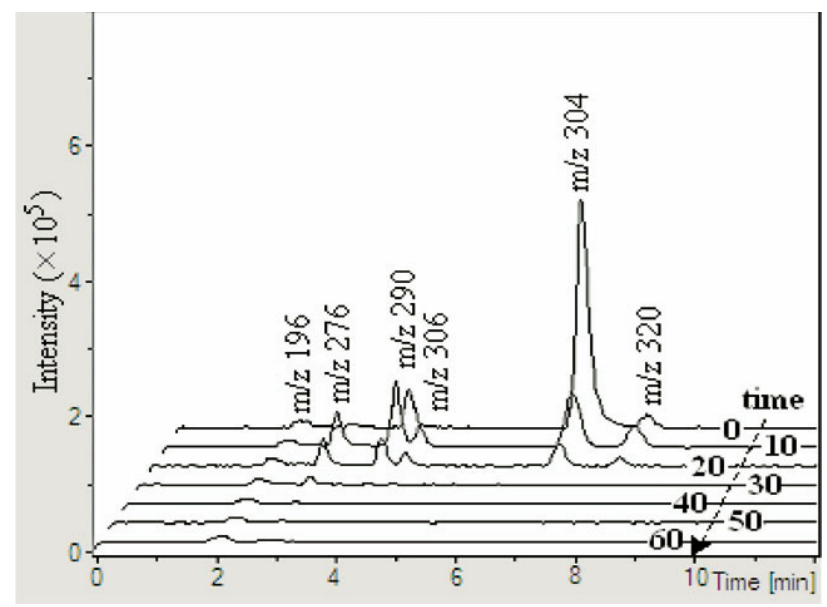

Figure 4. Chromatograms monitored in full scan MS corresponding to the solutions of methyl orange after being degraded for $0,10,20,30,40,50$, and $60 \mathrm{~min}$. Each peak is characterized by its $m / z$ value. 

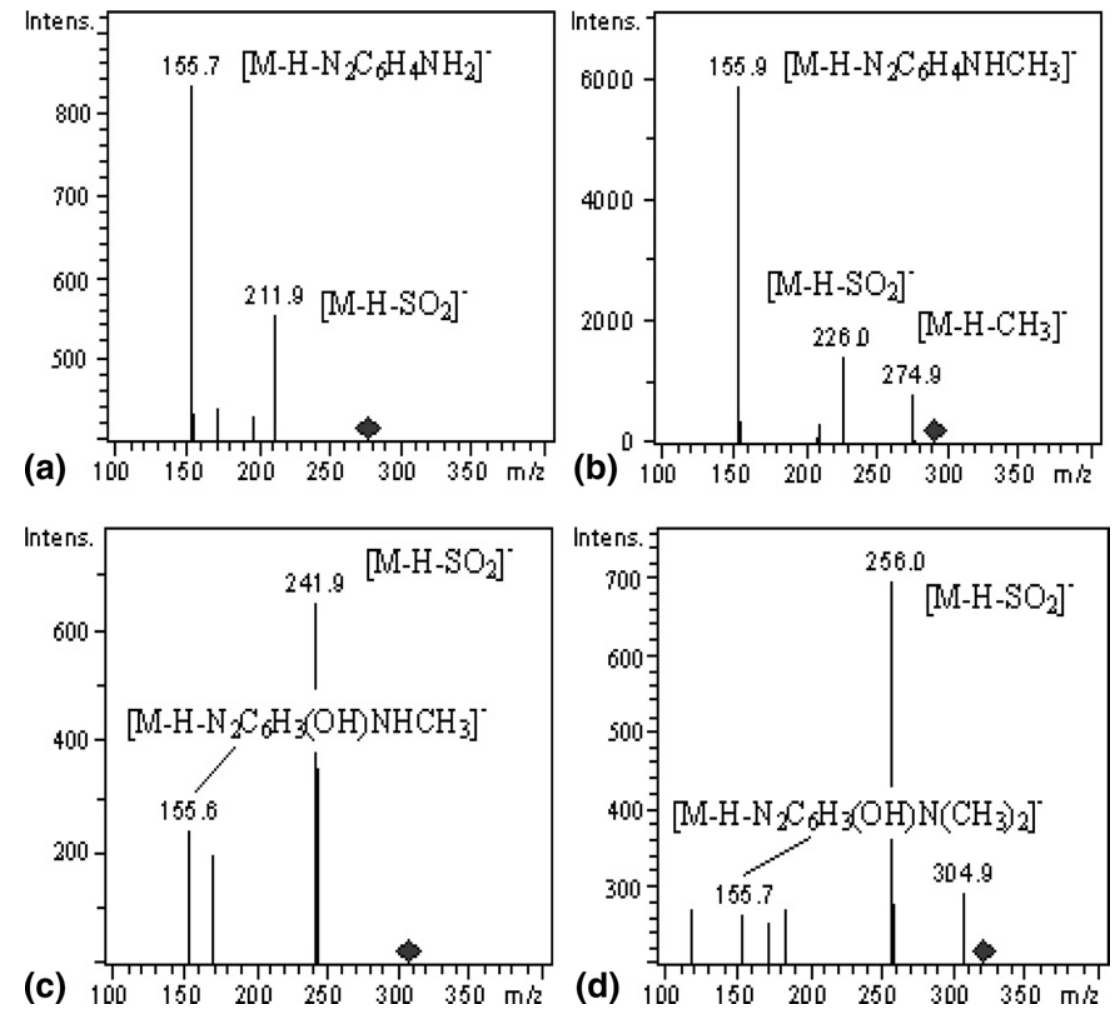

Figure 5. MS/MS spectrum of methyl orange degradation products. (a) MS/MS spectrum of $m / z 276$; (b) MS/MS spectrum of $\mathrm{m} / \mathrm{z}$ 290; (c) MS/MS spectrum of $\mathrm{m} / \mathrm{z}$ 306; (d) MS/MS spectrum of $\mathrm{m} / \mathrm{z} 320$.

\section{Mechanism of Photocatalysis}

It has reported that the oxygen defects and/or metal nanoparticles on the surface of $\mathrm{ZnO}$ nanorods would improve the separation of photogenerated electronhole pairs, and thus enhance the photocatalytic activity of ZnO-based photocatalysts [12, 15]. Figure 7 shows

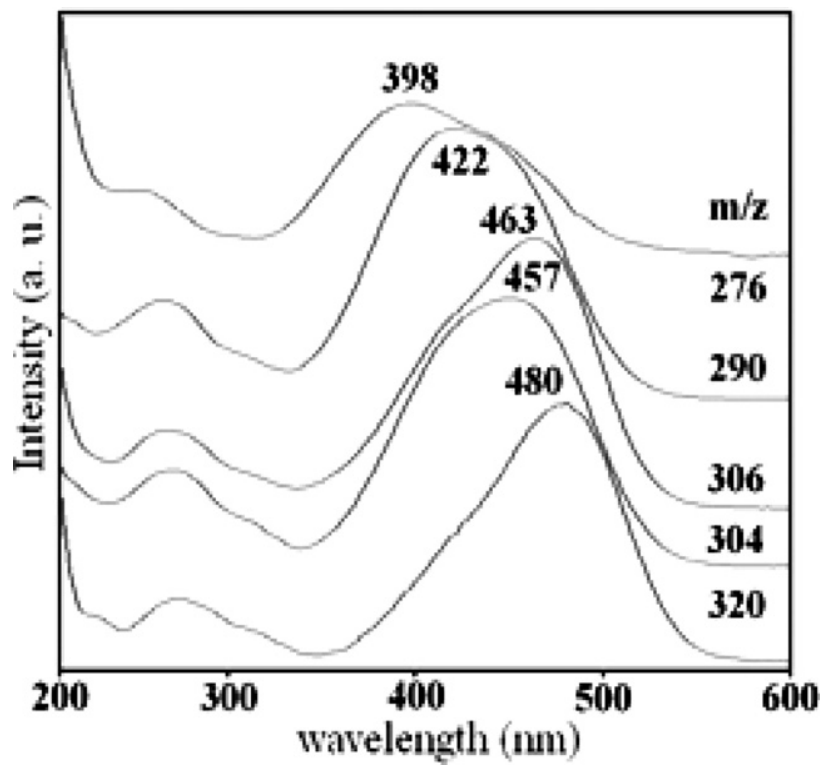

Figure 6. UV-vis absorption spectra for the species of $\mathrm{m} / \mathrm{z} 276$, $290,306,304$, and 320. the proposed band structure and photocatalytic mechanism of the $\mathrm{Ag} / \mathrm{ZnO}$ photocatalyst; the photocatalytic reaction process can be proposed as follows $[28,29]$ :

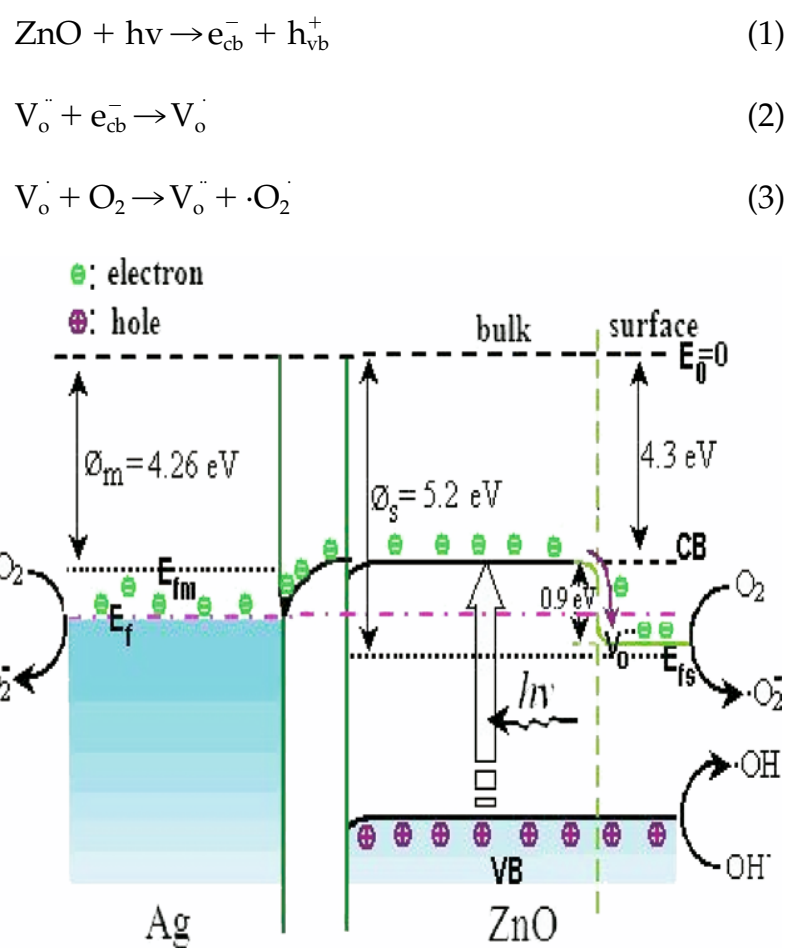

Figure 7. Proposed band structure and photocatalytic mechanism of the synthesized $\mathrm{Ag} / \mathrm{ZnO}$ heterostructure nanocrystals. 


$$
\begin{aligned}
& \mathrm{Ag} \rightarrow \mathrm{Ag}^{+}+\mathrm{e}^{-}\left(\text {or } \mathrm{e}_{\mathrm{cb}}^{-}\right) \\
& \mathrm{e}^{-}\left(\text {or }_{\mathrm{cb}}^{-}\right)+\mathrm{O}_{2} \rightarrow \cdot \mathrm{O}_{2}^{-} \\
& \mathrm{e}_{\mathrm{cb}}^{-}+\mathrm{Ag}^{+} \rightarrow \mathrm{Ag} \\
& \mathrm{h}_{\mathrm{vb}}^{+}+\mathrm{OH}^{-} \rightarrow \cdot \mathrm{OH}
\end{aligned}
$$

On the basis of the preceding discussion, it can be concluded that: (1) ZnO nanorods serve as an electron and hole source (see eq 1) for degradation of organic dye; when semiconductor nanocrystals are irradiated by UV light with energy higher than or equal to the band gap of $\mathrm{ZnO}$ nanocrystals, an electron $\left(\mathrm{e}^{-}\right)$in the valence band $(\mathrm{VB})$ can be excited to the conduction band (CB) with simultaneous generation of a hole $\left(\mathrm{h}^{+}\right)$ in the VB; (2) oxygen vacancy defects (see $\mathrm{V}_{\mathrm{o}} \cdot$ and $\mathrm{V}_{\mathrm{o}}{ }^{*}$ in eqs 2 and 3) and Ag nanoparticles on the surface of $\mathrm{ZnO}$ nanorods act as a sink for the electrons (see eqs 4 and 6 ) and improve the separation of electron-hole pairs generated in eq 1; (3) the photoelectron can be easily trapped by electronic acceptors like adsorbed $\mathrm{O}_{2}$, to further produce a superoxide radical anion $\left(\cdot \mathrm{O}_{2}{ }^{-}\right.$) (see eq 5). The photoinduced holes can be easily trapped by $\mathrm{OH}^{-}$to further produce a hydroxyl radical species $(\cdot \mathrm{OH})$ (see eq 7$)$; the generated superoxide anion radical $\left(\cdot \mathrm{O}_{2}{ }^{-}\right)$and hydroxyl radical species $(\cdot \mathrm{OH})$ determine the overall photocatalytic reaction; for example, $\cdot \mathrm{OH}$ is an extremely strong oxidant for the partial or complete mineralization of organic chemicals [30].

On the basis of the preceding experimental results, we propose the chemical structure of the photocatalytic degradation products for $\mathrm{MO}$ (see Figure 8). Four major compounds appeared during the photocatalytic process. They come from the demethylation and hydroxylation of $\mathrm{MO}$.

\section{Conclusions}

$\mathrm{Ag} / \mathrm{ZnO}$ nanocatalysts with excellent photocatalytic performance were successfully prepared using a simple solvothermal method in this study, which has shown that photocatalytic degradation of $\mathrm{MO}$ can be readily performed in the presence of $\mathrm{Ag} / \mathrm{ZnO}$ in an aqueous suspension system, leading to the complete

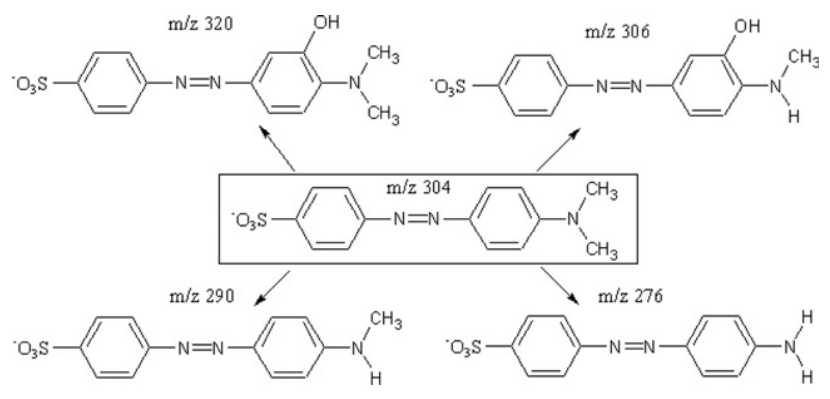

Figure 8. Proposed degradation products of methyl orange. mineralization of the dye. The required time for an entire degradation of $\mathrm{MO}$ using $\mathrm{Ag} / \mathrm{ZnO}$ catalysts is less than $60 \mathrm{~min}$, much shorter than the corresponding value by using other catalysts. The structures of the intermediates formed during the photocatalytic process are based mainly on demethylation and hydroxylation.

\section{Acknowledgments}

This work is financially supported by the National Nature Sciences Funding of China (20735002, 20575011), the Key Natural Sciences Funding of Fujian Province, China (D0520001), and the Key Science and Technology project of Fujian Province, China (2003Y005).

\section{References}

1. Qaradawi, S. A.; Salman, S. R. Photocatalytic Degradation of Methyl Orange as a Model Compound. J. Photochem. Photobiol. A: Chem. 2002, 148, 161-168.

2. Wang, C.; Wang, X. M.; Xu, B. Q.; Zhao, J. C.; Mai, B. X.; Peng, P. A.; Sheng, G. Y.; Fu, J. M. Enhanced Photocatalytic Performance of Nanosized Coupled $\mathrm{ZnO} / \mathrm{SnO}_{2}$ Photocatalysts for Methyl Orange Degradation. J. Photochem. Photobiol. A: Chem. 2004, 168, 47-52.

3. Liu, S.; Yang, J. H.; Choy, J. H. Microporous $\mathrm{SiO}_{2}-\mathrm{TiO}_{2}$ Nanosols Pillared Montmorillonite for Photocatalytic Decomposition of Methyl Orange. J. Photochem. Photobiol. A: Chem. 2006, 179, 75-80.

4. Baiocchi, C.; Brussino, M. C.; Pramauro, E.; Bianco-Prevot, A.; Palmisano, L.; Marci, G. Characterization of Methyl Orange and Its Photocatalytic Degradation Products by HPLC/UV-VIS Diode Array and Atmospheric Pressure Ionization Quadrupole Ion-trap Mass Spectrometry. Int. J. Mass Spectrom. 2002, 214, 247-256.

5. Kansal, S. K.; Singh, M.; Sud, D. Studies on Photodegradation of Two Commercial Dyes in Aqueous Phase Using Different Photocatalysts. J. Hazard. Mater. 2007, 141, 581-590.

6. Bianco-Prevot, A.; Basso, A.; Baiocchi, C.; Pazzi, M.; Marci, G.; Augugliaro, V.; Palmisano, L.; Pramauro, E. Analytical Control of Photocatalytic Treatments: Degradation of a Sulfonated Azo Dye. Anal. Bioanal. Chem. 2004, 378, 214-220.

7. Liu, Y.; Chen, X.; Li, J.; Burda, C. Photocatalytic Degradation of Azo Dyes by Nitrogen-Doped $\mathrm{TiO}_{2}$ Nanocatalysts. Chemosphere 2005, 61, 11-18.

8. Mansilla, H.; Villaseñor, J.; Maturana, G.; Baeza, J.; Freer, J.; Durán, N. ZnO-Catalysed Photodegradation of Kraft Black Liquor. J. Photochem. Photobiol. A: Chem. 1994, 78, 267-273.

9. Ohnishi, H.; Matsumura, M.; Tsubomura, H.; Iwasaki, M. Bleaching of Lignin Solution by a Photocatalyzed Reaction on Semiconductor Photocatalysts. Ind. Eng. Chem. Res. 1989, 28, 719-724.

10. Peralta-Zamora, P.; Demoraes, S. G.; Pelegrini, R.; Freire, M.; Reyes, J.; Mansilla, H.; Duran, N. Evaluation of $\mathrm{ZnO}, \mathrm{TiO}_{2}$ and Supported $\mathrm{ZnO}$ on the Photoassisted Remediation of Black Liquor, Cellulose and Textile Mill Effluents. Chemosphere 1998, 36, 2119-2133.

11. Richard, C.; Bosquet, F.; Pilichowski, J. F. Photocatalytic Transformation of Aromatic Compounds in Aqueous Zinc Oxide Suspensions: Effect of Substrate Concentration on the Distribution of Products. J. Photochem. Photobiol. A: Chem. 1997, 108, 45-49.

12. Wood, A.; Giersig, M.; Mulvaney, P. Fermi Level Equilibration in Quantum Dot-Metal Nanojunctions. J. Phys. Chem. B 2001, 105, 8810 8815.

13. Stroyuk, A. L.; Shvalagin, V. V.; Kuchmii, S. Y. Photochemical Synthesis and Optical Properties of Binary and Ternary Metal-Semiconductor Composites Based on Zinc Oxide Nanoparticles. J. Photochem. Photobiol. A: Chem. 2005, 173, 185-194.

14. Height, M. J.; Pratsinis, S. E.; Mekasuwandumrong, O.; Praserthdam, P. $\mathrm{Ag}-\mathrm{ZnO}$ Catalysts for UV-Photodegradation of Methylene Blue. Appl. Catal. B 2006, 63, 305-312.

15. Gouvêa, C.; Wypych, F.; Moraes, S.; Durán, N.; Peralta-Zamora, P. Semiconductor-Assisted Photodegradation of Lignin, Dye, and Kraft Effluent by Ag-Doped ZnO. Chemosphere 2000, 40, 427-432.

16. Ansorgova, D.; Holcapek, M.; Jandera, P. Ion-Pairing High-Performance Liquid Chromatography-Mass Spectrometry of Impurities and Reduction Products of Sulphonated Azo Dyes. J. Sep. Sci. 2003, 26, 1011-1027.

17. Plum, A.; Rehorek, A. Strategies for Continuous On-line High Performance Liquid Chromatography Coupled with Diode Array Detection and Electrospray Tandem Mass Spectrometry for Process Monitoring of Sulphonated Azo Dyes and Their Intermediates in Anaerobic-Aerobic Bioreactors. J. Chromatogr. A 2005, 1084, 119-133.

18. Gosetti, F.; Gianotti, V.; Angioi, S.; Polati, S.; Marengo, E.; Gennaro, M. C. Oxidative Degradation of Food Dye E133 Brilliant Blue FCF 
Liquid Chromatography-Electrospray Mass Spectrometry Identification of the Degradation Pathway. I. Chromatogr. A 2004, 1054, 379-387.

19. Gosetti, F.; Gianotti, V.; Polati, S.; Gennaro, M. C. HPLC-MS Degradation Study of E1 10 Sunset Yellow FCF in a Commercial Beverage. J. Chromatogr. A 2005, 1090, 107-115.

20. Gosetti, F.; Gianotti, V.; Ravera, M.; Gennaro, M. C. HPLC-MS ${ }^{\mathrm{n}}$ to Investigate the Oxidative Destruction Pathway of Aromatic Sulfonate Wastes. J. Environ. Qual. 2005, 34, 2328-2333.

21. Zheng, Y.; Zheng, L.; Zhan, Y.; Lin, X.; Zheng, Q.; Wei, K. Ag/ZnO Heterostructure Nanocrystals: Synthesis, Characterization, and Photocatalysis. Inorg. Chem. 2007, 46, 6980-6986.

22. Holčapek, M.; Volná, K.; Vanřková, D. Effects of Functional Groups on the Fragmentation of Dyes in Electrospray and Atmospheric pressure Chemical Ionization Mass Spectra. Dyes Pigments 2007, 75, 156-165.

23. Bejarano-Pérez, N. J.; Suárez-Herrera, M. F. Sonophotocatalytic Degradation of Congo Red and Methyl Orange in the Presence of $\mathrm{TiO}_{2}$ as a Catalyst. Ultrasonics Sonochem. 2007, 14, 589-595.

24. Peral, J.; Mills, A. Factors Affecting the Kinetics of Methyl Orange Reduction Photosensitized by Colloidal CdS. J. Photochem. Photobiol. A: Chem. 1993, 73, 47-52.
25. Badr, Y.; Mahmoud, M. A. Photocatalytic Degradation of Methyl Orange by Gold Silver Nano-Core/Silica Nano-Shell. J. Phys. Chem. Solids 2007, 68, 413-419.

26. Ge, L.; Xu, M. X.; Fang, H. B. Photo-Catalytic Degradation of Methyl Orange and Formaldehyde by $\mathrm{Ag} / \mathrm{InVO}_{4}-\mathrm{TiO}_{2}$ Thin Films under Visible-Light Irradiation. J. Mol. Catal. A: Chem. 2006, 258, 68-76.

27. Wang, X. H.; Li, J. G.; Kamiyama, H.; Moriyoshi, Y.; Ishigaki, T. Wavelength-Sensitive Photocatalytic Degradation of Methyl Orange in Aqueous Suspension over Iron(III)-Doped $\mathrm{TiO}_{2}$ Nanopowders under UV and Visible Light Irradiation. J. Phys. Chem. B 2006, 110, 6804-6809.

28. Zheng, Y, Chen, C.; Zhan, Y.; Lin, X.; Zheng, Q.; Wei, K.; Zhu, J; Zhu, Y. Luminescence and Photocatalytic Activity of ZnO Nanocrystals: Correlation between Structure and Property. Inorg. Chem. 2007, 46, 6675-6682

29. Wang, X.; Kong, X.; Yu, Y.; Zhang, H. Synthesis and Characterization of Water-Soluble and Bifunctional ZnO-Au Nanocomposites. J. Phys. Chem. C 2007, 111, 3836-3841.

30. Yatmaz, H. C.; Akyol, A.; Bayramoglu, M. Kinetics of the Photocatalytic Decolorization of an Azo Reactive Dye in Aqueous $\mathrm{ZnO}$ Suspensions. Ind. Eng. Chem. Res. 2004, 43, 6035-6039. 